\author{
M.T. Jenaliyev ${ }^{1}$, M.G. Yergaliyev ${ }^{1}$, A.A. Assetov ${ }^{2}{ }^{*}$, A.K. Kalibekova $^{1}$ \\ ${ }^{1}$ Institute of Mathematics and Mathematical Modeling, Almaty, Kazakhstan; \\ ${ }^{2}$ Karagandy University of the name of academician E.A. Buketov, Karaganda, Kazakhstan \\ (E-mail: muvasharkhan@gmail.com,ergaliev.madi.g@gmail.com, \\ bekaaskar@mail.ru,kalibekovaaidana2014@gmail.com)
}

\title{
On One Initial Boundary Value Problem for the Burgers Equation in a Rectangular Domain
}

\begin{abstract}
We consider some initial boundary value problems for the Burgers equation in a rectangular domain, which in a sense can be taken as a model one. The fact is that such a problem often arises when studying the Burgers equation in domains with moving boundaries. Using the methods of functional analysis, priori estimates, and Faedo-Galerkin in Sobolev spaces and in a rectangular domain, we show the correctness of the initial boundary value problem for the Burgers equation with nonlinear boundary conditions of the Neumann type.
\end{abstract}

Keywords: Burgers equation, boundary value problem, Sobolev classes, rectangular domain, Galerkin methods, priori estimates.

\section{Introduction}

The study of the Burgers equation has a long history, some of which is given in [1-4], as well as in monographs [5] and [6].

In works [1] and [2] in Sobolev spaces, the correctness of the boundary value problem for the Burgers equation was established. In this case, the domain of independent variables degenerated according to a nonlinear law, and homogeneous Dirichlet conditions were set on the boundary.

The infiltration of the wetting front into a porous medium is a classical problem with a free boundary. Historically, the first example is the Green-Ampt model for water flow in soils [7]. There is a huge variety of situations (chemically reacting media, deformable media, capillarity effects, mass transfer, mixture flows, media with a complex structure, pollution, reclamation, soil freezing, production of composite materials, brewing, etc.).

Nonlinear Burgers equations and their modifications are also suitable models of fluid motion in porous media [8-13].

The range of application of boundary value problems for parabolic equations in a domain with a boundary that changes over time is quite wide. Such problems arise in the study of thermal processes in electrical contacts [14], the processes of ecology and medicine [15], in solving some problems of hydromechanics [16], thermomechanics in thermal shock [17], and so on.

In this paper, in Sobolev classes, we study the solvability of the initial boundary value problem for the Burgers equation in a rectangular domain with nonlinear boundary conditions of the Neumann type.

In Section 1, the statement of the boundary value problem under study is given, and the main result of the work is formulated. We study the questions of unique solvability of two auxiliary boundary value problems for the Burgers equation in rectangular and non-rectangular domains, which are used in the proof of the main results of the work. Sections 2-5 are devoted to the first auxiliary problem. In these sections, the correctness of this problem in Sobolev classes is established by the methods of a priori estimates and Faedo-Galerkin. In sections $4-5$, Theorem 1.1, the main result of the work is proved. The work is completed by a brief conclusion.

\footnotetext{
* Corresponding author.

E-mail:bekaaskar@mail.ru
} 


\section{Problem statement and main result}

In the domain $Q_{y t}=\{y, t \mid y \in(0,1), t \in(0, T)\}$, we consider the following initial boundary value problem:

$$
\begin{gathered}
\partial_{t} w+\alpha(t) w \partial_{y} w-\beta(t) \partial_{y}^{2} w+\gamma(y, t) \partial_{y} w=g \\
{\left.\left[\frac{\alpha(t)}{3} w^{2}-\beta(t) \partial_{y} w\right]\right|_{y=0}=0,\left.\left[\frac{\alpha(t)}{3} w^{2}-\beta(t) \partial_{y} w\right]\right|_{y=1}=0,0<t<T} \\
w(y, 0)=0,0<y<1 .
\end{gathered}
$$

where the given continuous functions $\alpha(t), \beta(t), \gamma(y, t)$ satisfy the following conditions

$$
\alpha^{\prime}(t) \leq 0, \beta^{\prime}(t) \leq 0, \alpha_{1} \leq \alpha(t) \leq \alpha_{2}, \beta_{1} \leq \beta(t) \leq \beta_{2},|\gamma(y, t)| \leq \gamma_{1},\left|\partial_{y} \gamma(y, t)\right| \leq \gamma_{1}, \forall t \in[0, T],
$$

with given positive constants

$$
\alpha_{i}, \beta_{i}, i=1,2, \gamma_{1} ; \alpha(t), \beta(t) \in C^{1}([0, T]), \partial_{y} \gamma(y, t) \in C\left(\bar{Q}_{y t}\right) .
$$

Theorem 1.1 (Main result). Let $g \in L_{2}\left(Q_{y t}\right)$ and conditions (1.4)-(1.5) be satisfied. Then boundary value problem (1.1)-(1.3) has a unique solution

$$
w \in H^{2,1}\left(Q_{y t}\right) \equiv L_{2}\left(0, T ; H^{2}(0,1)\right) \cap H^{1}\left(0, T ; L_{2}(0,1)\right) .
$$

To apply the Faedo-Galerkin method, we need to solve the following spectral problem:

$$
\begin{gathered}
-Y^{\prime \prime}(y)=\lambda^{2} Y(y), y \in(0,1), \\
Y^{\prime}(0)+\lambda^{2} Y(0)=0, \\
Y^{\prime}(1)-\lambda^{2} Y(1)=0,
\end{gathered}
$$

obtained by applying the variable separation method $(u(y, t)=F(t) Y(y))$ from the following problem

$$
\begin{gathered}
\partial_{t} u-\partial_{y}^{2} u=0, y \in(0,1), t \in(0, T), \\
\partial_{t} u-\left.\partial_{x} u\right|_{y=0}=0, \quad \partial_{t} u+\left.\partial_{x} u\right|_{y=1}=0, \\
u(y, 0)=u_{0}(y) .
\end{gathered}
$$

2 Solving spectral problem (1.6)-(1.8)

We seek the general solution to equation (1.6) in the form

$$
Y(y)=C_{1} \exp \{i \lambda y\}+C_{2} \exp \{-i \lambda y\}, i=\sqrt{-1} .
$$

Satisfying (2.1) to boundary conditions (1.7)-(1.8), we obtain

$$
\begin{gathered}
Y_{01}(y)=1, \lambda_{01}=0, \tan \frac{\lambda_{01}}{2}=-\lambda_{01}, \\
Y_{2 n-1}(y)=\cos \frac{\lambda_{2 n-1}(1-2 y)}{2}, \lambda_{2 n-1}=(2 n-1) \pi+\varepsilon_{2 n-1}, \tan \frac{\lambda_{2 n-1}}{2}=-\lambda_{2 n-1}, n \in \mathbb{N}, \\
Y_{02}(y)=\sin \frac{\lambda_{02}(1-2 y)}{2}, \lambda_{02} \approx \frac{2 \pi}{5}, \cot \frac{\lambda_{02}}{2}=\lambda_{02}, \\
Y_{2 n}(y)=\sin \frac{\lambda_{2 n}(1-2 y)}{2}, \lambda_{2 n}=2 n \pi+\varepsilon_{2 n}, \cot \frac{\lambda_{2 n}}{2}=\lambda_{2 n}, n \in \mathbb{N} .
\end{gathered}
$$

It is easy to see that the solutions of equations

$$
\tan \frac{\lambda_{2 n-1}}{2}=-\lambda_{2 n-1}, n \in \mathbb{N}, \quad \text { and } \quad \cot \frac{\lambda_{2 n}}{2}=\lambda_{2 n}, n \in \mathbb{N},
$$


are, respectively, close to points $(2 n-1) \pi$ and $2 n \pi, n \in \mathbb{N}$, and with the growth of $n$ they approach arbitrarily close from the right to the corresponding specified points $(2 n-1) \pi$ and $2 n \pi, n \in \mathbb{N}$, i.e. $\varepsilon_{n} \rightarrow 0+$ at $n \rightarrow \infty$ (see Figure 2.1-2.2). If we introduce the notation $2 x=(1-2 y) \pi$, then we get: $x \in(-\pi / 2, \pi / 2)$.

By the Paley-Wiener theorem ([18], chapter V, 86, example), the system of functions (2.2) and (2.3) is complete in $L_{2}(0,1)$, since the system of functions:

$$
\frac{\sqrt{2} \cos x}{\sqrt{\pi}}, \frac{\sqrt{2} \sin 2 x}{\sqrt{\pi}}, \frac{\sqrt{2} \cos 3 x}{\sqrt{\pi}}, \frac{\sqrt{2} \sin 4 x}{\sqrt{\pi}}, \ldots
$$

which is complete in $L_{2}(-\pi / 2, \pi / 2)$, will differ little from it. For the latter system, it is sufficient to make the replacement $x_{1}=x+\pi / 2$. We get the system of sines:

$$
\frac{\sqrt{2} \sin x_{1}}{\sqrt{\pi}}, \frac{\sqrt{2} \sin 2 x_{1}}{\sqrt{\pi}}, \frac{\sqrt{2} \sin 3 x_{1}}{\sqrt{\pi}}, \frac{\sqrt{2} \sin 4 x_{1}}{\sqrt{\pi}}, \ldots
$$

which is complete in $L_{2}(0, \pi)$.

Note that the system of functions (2.2) and (2.3) is not orthogonal in $L_{2}(0,1)$.

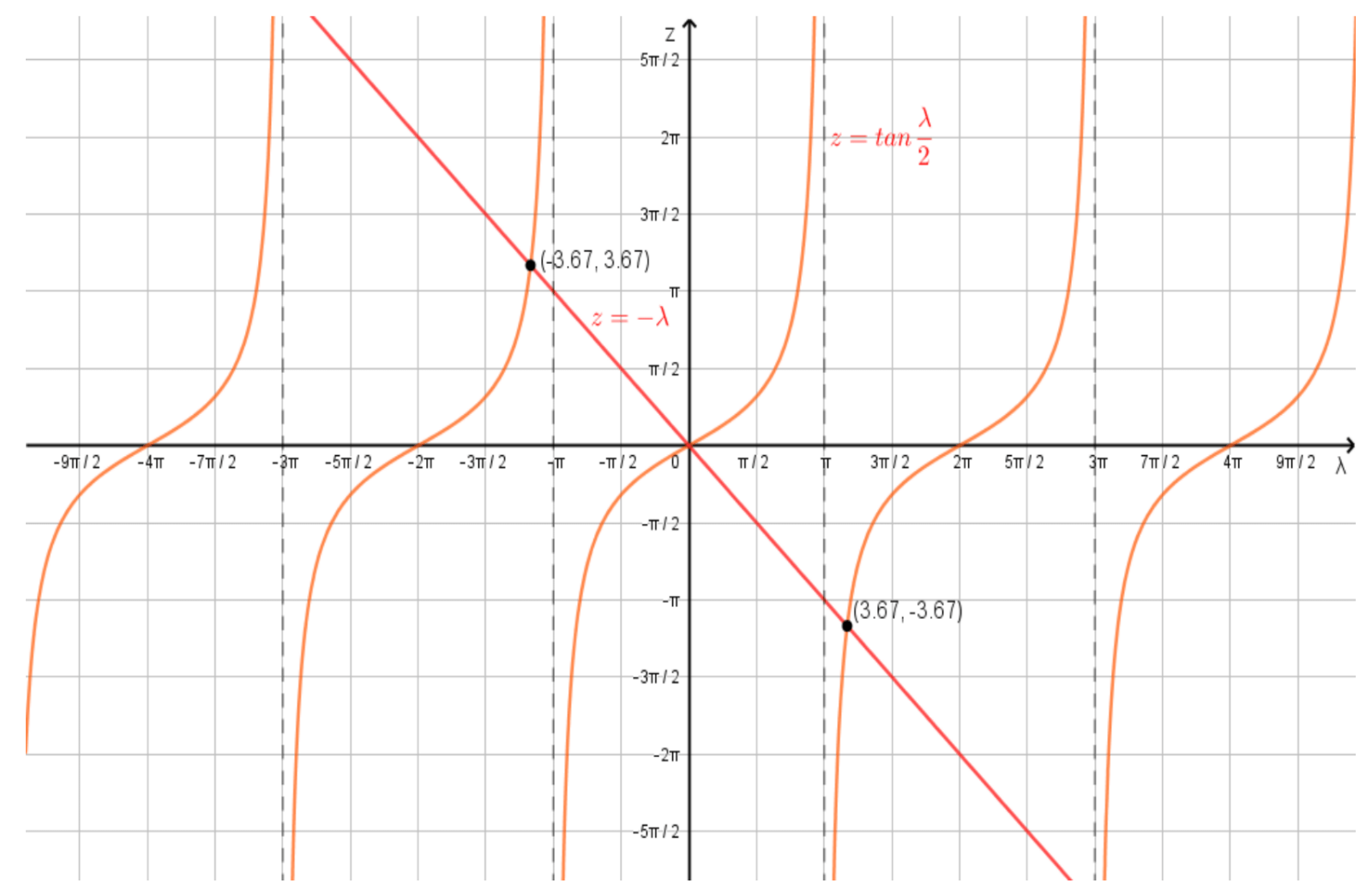

Figure 2.1. Graphs of functions $z=-\lambda, z=\tan \frac{\lambda}{2}$

Remark 2.1. The applicability of the Paley-Wiener theorem ([18], chapter V, 86, example) follows from the relations:

$$
\lambda_{1} \approx 3.673, \quad \lambda_{1}-\pi \approx 0.533, \quad M \pi=\left|\lambda_{1}-\pi\right|<0.54<\ln 2 \approx 0.693, \quad \theta=\exp \{M \pi\}-1<1
$$




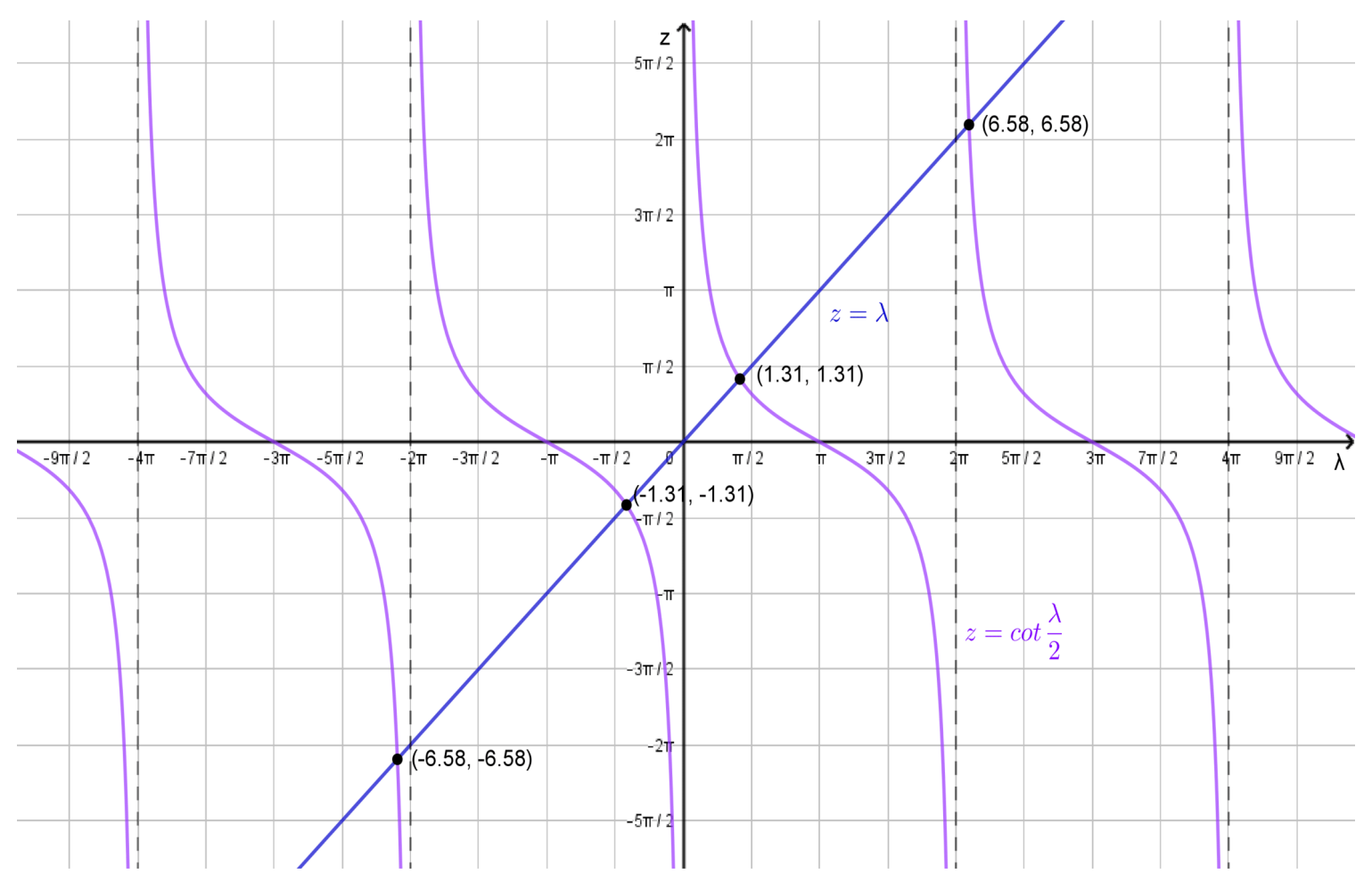

Figure 2.2. Graphs of functions $z=\lambda, z=\cot \frac{\lambda}{2}$

\section{Setting and solving the approximate problem}

We multiply equation (1.1) scalarly in $L_{2}(0,1)$ by function $v \in H^{1}(0,1)$. As a result, taking into account initial (1.3) and boundary conditions (1.2) we will have a weak statement of problem (1.1)-(1.3):

$$
\begin{gathered}
\int_{0}^{1} \partial_{t} w v d y+\alpha(t) \int_{0}^{1} w \partial_{y} w v d y+\beta(t) \int_{0}^{1} \partial_{y} w \partial_{y} v d y+\int_{0}^{1} \gamma(y, t) \partial_{y} w v d y- \\
-\frac{\alpha(t)}{3} w^{2}(1, t) v(1, t)+\frac{\alpha(t)}{3} w^{2}(0, t) v(0, t)=\int_{0}^{1} g v d y, \quad \forall v \in H^{1}(0,1), \\
w(y, 0)=0, \quad y \in(0,1) .
\end{gathered}
$$

We introduce the following approximate solution

$$
w_{n}(y, t)=\sum_{j=1}^{n} c_{j}(t) Y_{j}(y), \quad w_{n}(y, 0)=\sum_{j=1}^{n} c_{j}(0) Y_{j}(y) .
$$

Next, we will satisfy this solution to an approximate version of problem (3.1)-(3.2):

$$
\begin{gathered}
\int_{0}^{1} \partial_{t} w_{n} Y_{j} d y+\alpha(t) \int_{0}^{1} w_{n} \partial_{y} w_{n} Y_{j} d y+\beta(t) \int_{0}^{1} \partial_{y} w_{n} \partial_{y} Y_{j} d y+\int_{0}^{1} \gamma(y, t) \partial_{y} w_{n} Y_{j} d y- \\
-\frac{\alpha(t)}{3} w_{n}^{2}(1, t) Y_{j}(1)+\frac{\alpha(t)}{3} w_{n}^{2}(0, t) Y_{j}(0)=\int_{0}^{1} g Y_{j} d y
\end{gathered}
$$




$$
w_{n}(y, 0)=0, \quad y \in(0,1)
$$

for all $j=0,1, \ldots, n$, and $t \in[0, T]$.

Lemma 3.1. Problem (3.4)-(3.5) has a unique solution $w_{n}(y, t)$.

Proof. Since the system of functions $Y_{1}(y), Y_{2}(y), \ldots$ is a basis in $L_{2}(0,1)$, we have

$$
\operatorname{det}\left\{W_{n}\right\}=\left\|\left(Y_{k}(y), Y_{j}(y)\right)\right\|_{k, j=1}^{n} \neq 0, \forall \text { finite } n ;
$$

$W_{n}$ is a Gram matrix, $(\cdot, \cdot)$ is the scalar product in $L_{2}(0,1), A_{n}=\left(\partial_{y} Y_{k}(y), \partial_{y} Y_{j}(y)\right)_{k, j=1}^{n}$,

$$
w_{n}^{2}(1, t) Y_{j}(1, t)-w_{n}^{2}(0, t) Y_{j}(0, t)=\left[\sum_{k=1}^{n} c_{k}(t) Y_{k}(1)\right]^{2} Y_{j}(1)-\left[\sum_{k=1}^{n} c_{k}(t) Y_{k}(0)\right]^{2} Y_{j}(0) .
$$

Further, if we introduce the notation

$$
\begin{aligned}
G_{n}(t)=\left\{g_{0}(t), \ldots, g_{n}(t)\right\}, P_{n}(t) & =\left\{p_{0}(t), \ldots, p_{n}(t)\right\}, H_{n}(t)=\left\{h_{0}(t), \ldots, h_{n}(t)\right\}, \\
C_{n}(t) & =\left\{c_{1}(t), \ldots, c_{n}(t)\right\},
\end{aligned}
$$

where

$$
\begin{gathered}
g_{j}(t)=\int_{0}^{1} g Y_{j}(y) d y, \quad p_{j}(t)=-\alpha(t) \int_{0}^{1} w_{n} \partial_{y} w_{n} Y_{j}(y) d y-\int_{0}^{1} \gamma(y, t) \partial_{y} w_{n}(y, t) Y_{j}(y) d y, \\
h_{j}(t)=\frac{\alpha(t)}{3}\left[\sum_{k=1}^{n} c_{k}(t) Y_{k}(1)\right]^{2} Y_{j}(1)-\left[\sum_{k=1}^{n} c_{k}(t) Y_{k}(0)\right]^{2} Y_{j}(0),
\end{gathered}
$$

for all $j=0,1, \ldots, n$, then problem (3.4)-(3.5) is equivalent to the following Cauchy problem for a finite system of nonlinear ordinary differential equations

$$
C_{n}^{\prime}(t)=W_{n}^{-1}\left[-\beta(t) A_{n} c(t)+P_{n}(t)+H_{n}(t)+G_{n}(t)\right], \quad C_{n}(0)=0 .
$$

Note that functions $p_{j}(t), h_{j}(t)$ are well-defined, and function $g_{j}(t)$ is square integrable (by virtue of $g \in L_{2}(Q)$ ). Theorefore, the Cauchy problem (3.6) is uniquely solvable on some interval $\left[0, T^{\prime}\right]$, where $T^{\prime} \leq T$. However, according to the priori estimates established below, we find that this solution $C_{n}(t)$ continues to a finite time $T$.

Thus, we find the functions $C_{n}(t)=\left\{c_{j}(t), j=0,1, \ldots, n\right\}$ as a solution to the Cauchy problem (3.6) for each fixed finite $n$, and together with them the only approximate solution $w_{n}(y, t)$ to problem (3.4)-(3.5). Lemma 3.1 is completely proved.

\section{A priori estimates}

Lemma 4.1. There exists a positive constant $K_{1}$ independent of $n$, such that for all $t \in[0, T]$ the following estimate takes place

$$
\left\|w_{n}(y, t)\right\|_{L_{2}(0,1)}^{2}+\beta_{1} \int_{0}^{t}\left\|\partial_{y} w_{n}(y, \tau)\right\|_{L_{2}(0,1)}^{2} d \tau \leq K_{1} .
$$

Proof. Multiplying (3.4) by $c_{j}(t)$, summing the result over $j$ from 1 to $n$ and using the equality

$$
\int_{0}^{1} w_{n}(y, t) \partial_{y} w_{n}(y, t) w_{n}(y, t) d y=\frac{1}{3} w_{n}^{3}(1, t)-\frac{1}{3} w_{n}^{3}(0, t),
$$

we obtain

$$
\frac{1}{2} \frac{d}{d t} \int_{0}^{1}\left|w_{n}(y, t)\right|^{2} d y+\beta(t) \int_{0}^{1}\left|\partial_{y} w_{n}(y, t)\right|^{2} d y=
$$




$$
=-\int_{0}^{1} \gamma(y, t) \partial_{y} w_{n}(y, t) w_{n}(y, t) d y+\int_{0}^{1} g(y, t) w_{n}(y, t) d y .
$$

Now, by integrating (4.1) with respect to $t$ from 0 to $t$ and using Cauchy's inequality

$$
\begin{gathered}
-\int_{0}^{1} \gamma(y, t) \partial_{y} w_{n}(y, t) w_{n}(y, t) d y \leq \frac{\beta_{1}}{2}\left\|\partial_{y} w_{n}(y, t)\right\|_{L_{2}(0,1)}^{2}+\frac{\gamma_{1}^{2}}{2 \beta_{1}}\left\|w_{n}(y, t)\right\|_{L_{2}(0,1)}^{2}, \\
\int_{0}^{1} g(y, t) w_{n}(y, t) d y \leq \frac{1}{2}\|g(y, t)\|_{L_{2}(0,1)}^{2}+\frac{1}{2}\left\|w_{n}(y, t)\right\|_{L_{2}(0,1)}^{2},
\end{gathered}
$$

we get

$$
\begin{gathered}
\left\|w_{n}(y, t)\right\|_{L_{2}(0,1)}^{2}+\beta_{1} \int_{0}^{t}\left\|\partial_{y} w_{n}(y, \tau)\right\|_{L_{1}(0,1)}^{2} d \tau \leq \\
\leq\left(\frac{\gamma_{1}^{2}}{\beta_{1}}+1\right) \int_{0}^{t}\left\|w_{n}(y, \tau)\right\|_{L_{2}(0,1)}^{2} d \tau+\int_{0}^{T}\|g(y, \tau)\|_{L_{2}(0,1)}^{2} d \tau .
\end{gathered}
$$

From (4.2) follows

$$
\left\|w_{n}(y, t)\right\|_{L_{2}(0,1)}^{2} \leq\left(\frac{\gamma_{1}^{2}}{\beta_{1}}+1\right) \int_{0}^{t}\left\|w_{n}(y, \tau)\right\|_{L_{2}(0,1)}^{2} d \tau+\int_{0}^{T}\|g(y, \tau)\|_{L_{2}(0,1)}^{2} d \tau .
$$

By applying the Gronwall's inequality, we obtain the estimate for $\left\|w_{n}(y, t)\right\|_{L_{2}(0,1)}^{2}$. By using this estimate in (4.2), we establish the required estimate for Lemma 4.1.

Embedding $H^{1}(0,1) \hookrightarrow C([0,1])$ from Lemma 4.1 we directly obtain:

Corollary 4.1. There exists a positive constant $K_{1}^{\prime}$ independent of $n$, such that for all $t \in[0, T]$ the following inequality holds

$$
\int_{0}^{t}\left|w_{n}(0, \tau)\right|^{2} d \tau+\int_{0}^{t}\left|w_{n}(1, \tau)\right|^{2} d \tau \leq 2 B \int_{0}^{t}\left\|w_{n}(y, t)\right\|_{H^{1}(0,1)}^{2} \leq K_{1}^{\prime},
$$

where $B$ is a constant of the embedding $H^{1}(0,1) \hookrightarrow C([0,1])$.

Lemma 4.2. For a positive constant $K_{2}$ independent of $n$, for all $t \in(0, T]$ the following inequality takes place:

$$
\left\|\partial_{y} w_{n}(y, t)\right\|_{L_{2}(0,1)}^{2}+\beta_{1} \int_{0}^{t}\left\|\partial_{y}^{2} w_{n}(y, \tau)\right\|_{L_{2}(0,1)}^{2} d \tau \leq K_{2} .
$$

Proof. Taking into account equality

$$
\sum_{j=1}^{n} c_{j} \lambda_{j}^{2} Y_{j}(y)=-\sum_{j=1}^{n} c_{j} \partial_{y}^{2} Y_{j}(y)=-\partial_{y}^{2} w_{n}(y, t),
$$

which follows from (1.6) and (3.3), and multiplying equality (3.4) by $c_{j} \lambda_{j}^{2}$ and summing over $j$ from 1 to $n$, we obtain

$$
\begin{gathered}
\frac{1}{2} \frac{d}{d t}\left\|\partial_{y} w_{n}(y, t)\right\|_{L_{2}(0,1)}^{2}+\beta(t)\left\|\partial_{y}^{2} w_{n}(y, t)\right\|_{L_{2}(0,1)}^{2}= \\
=\alpha(t)\left(w_{n}(y, t) \partial_{y} w_{n}(y, t), \partial_{y}^{2} w_{n}(y, t)\right)+\left(\gamma(y, t) \partial_{y} w_{n}(y, t), \partial_{y}^{2} w_{n}(y, t)\right)- \\
-\left(g(y, t), \partial_{y}^{2} w_{n}(y, t)\right)+\left.\partial_{t} w_{n}(y, t) \partial_{y} w_{n}(y, t)\right|_{y=0} ^{y=1}=
\end{gathered}
$$




$$
\begin{gathered}
=\alpha(t)\left(w_{n}(y, t) \partial_{y} w_{n}(y, t), \partial_{y}^{2} w_{n}(y, t)\right)+\left(\gamma(y, t) \partial_{y} w_{n}(y, t), \partial_{y}^{2} w_{n}(y, t)\right)- \\
-\left(g(y, t), \partial_{y}^{2} w_{n}(y, t)\right)+\left.\frac{\alpha(t)}{9 \beta(t)} \partial_{t}\left[w_{n}(y, t)\right]^{3}\right|_{y=0} ^{y=1}
\end{gathered}
$$

We will use the relations (similar ones are true for the term with $w_{n}(0, t)$ )

$$
\begin{aligned}
& \int_{0}^{t} \frac{\alpha(t)}{9 \beta(t)} \partial_{t}\left[w_{n}(1, t)\right]^{3} d t=\frac{\alpha(t)}{9 \beta(t)}\left[w_{n}(1, t)\right]^{3}-\int_{0}^{t} \frac{\alpha^{\prime}(t) \beta(t)-\alpha(t) \beta^{\prime}(t)}{9[\beta(t)]^{2}}\left[w_{n}(1, t)\right]^{3} d t \leq \\
& \leq \frac{\alpha_{2}}{9 \beta_{1}}\left|w_{n}(1, t)\right|^{3}+C_{1} \int_{0}^{t}\left|w_{n}(1, t)\right|^{3} d t, \quad \text { where } 9 C_{1} \beta_{1}^{2}=\max _{0 \leq t \leq T}\left|\alpha^{\prime}(t) \beta(t)-\alpha(t) \beta^{\prime}(t)\right| .
\end{aligned}
$$

Let us establish the following estimate

$$
\begin{gathered}
\frac{\alpha_{2}}{9 \beta_{1}}\left|w_{n}(1, t)\right|^{3} \leq\left\|w_{n}(y, t)\right\|_{L_{\infty}(0,1)}^{3} \leq \frac{\alpha_{2}}{9 \beta_{1}}\left\|w_{n}(y, t)\right\|_{H^{1}(0,1)}^{3 / 2}\left\|w_{n}(y, t)\right\|_{L_{2}(0,1)}^{3 / 2}= \\
=\frac{\alpha_{2}}{9 \beta_{1}}\left\|w_{n}(y, t)\right\|_{H^{1}(0,1)}^{3 / 2}\left\|w_{n}(y, t)\right\|_{L_{2}(0,1)}^{1 / 2}\left\|w_{n}(y, t)\right\|_{L_{2}(0,1)} .
\end{gathered}
$$

In the previous relation, we used the interpolation inequality from ([19], Theorems 5.8-5.9, p.140-141). Now, applying Young's inequality $\left(p^{-1}+q^{-1}=1\right)$ :

$$
|A B|=\left|\left(a^{1 / p} A\right)\left(a^{1 / q} \frac{B}{a}\right)\right| \leq \frac{a}{p}|A|^{p}+\frac{a}{q a^{q}}|B|^{q},
$$

where

$$
A=\left\|w_{n}(y, t)\right\|_{H^{1}(0,1)}^{3 / 2}, \quad B=\frac{\alpha_{2}}{9 \beta_{1}}\left\|w_{n}(y, t)\right\|_{L_{2}(0,1)}\left\|w_{n}(y, t)\right\|_{L_{2}(0,1)}^{1 / 2}, \quad a=\frac{1}{6}, \quad p=\frac{4}{3}, \quad q=4
$$

from here, we get

$$
\begin{gathered}
\frac{\alpha_{2}}{9 \beta_{1}}\left|w_{n}(1, t)\right|^{3} \leq \frac{1}{8}\left\|\partial_{y} w_{n}(y, t)\right\|_{L_{2}(0,1)}^{2}+\left[\frac{1}{8}+\frac{2 \alpha_{2}^{4}}{3^{5} \beta_{1}^{4}}\left\|w_{n}(y, t)\right\|_{L_{2}(0,1)}^{4}\right]\left\|w_{n}(y, t)\right\|_{L_{2}(0,1)}^{2} \leq \\
\leq \frac{1}{8}\left\|\partial_{y} w_{n}(y, t)\right\|_{L_{2}(0,1)}^{2}+D_{1},
\end{gathered}
$$

where the constant $D_{1}$ is determined according to the estimates of Lemma 4.1 and Corollary 4.1.

Similarly to the previous one, we obtain

$$
\begin{gathered}
\frac{\alpha_{2}}{9 \beta_{1}}\left|w_{n}(0, t)\right|^{3} \leq \frac{1}{8}\left\|\partial_{y} w_{n}(y, t)\right\|_{L_{2}(0,1)}^{2}+\left[\frac{1}{8}+\frac{2 \alpha_{2}^{4}}{3^{5} \beta_{1}^{4}}\left\|w_{n}(y, t)\right\|_{L_{2}(0,1)}^{4}\right]\left\|w_{n}(y, t)\right\|_{L_{2}(0,1)}^{2} \leq \\
\leq \frac{1}{8}\left\|\partial_{y} w_{n}(y, t)\right\|_{L_{2}(0,1)}^{2}+D_{0},
\end{gathered}
$$

where the constant $D_{0}$ is determined according to the estimates of Lemma 4.1 and Corollary 4.1.

First of all, we consider the estimates of the nonlinear summands from 4.4. To begin with, we have

$$
\begin{gathered}
\left|\left(w_{n}(y, t) \partial_{y} w_{n}(y, t), \partial_{y}^{2} w_{n}(y, t)\right)\right| \leq\left\|w_{n}(y, t)\right\|_{L_{4}(0,1)}\left\|\partial_{y} w_{n}(y, t)\right\|_{H^{1}(0,1)}\left\|\partial_{y} w_{n}(y, t)\right\|_{L_{4}(0,1)} \leq \\
\leq\left\|w_{n}(y, t)\right\|_{L_{4}(0,1)}\left\|\partial_{y} w_{n}(y, t)\right\|_{H^{1}(0,1)}\left\|\partial_{y} w_{n}(y, t)\right\|_{L_{\infty}(0,1)} .
\end{gathered}
$$

Further, consifeing the interpolation inequality from ([19], Theorems 5.8-5.9, p.140-141)

$$
\alpha_{2}\left\|\partial_{y} w_{n}(y, t)\right\|_{L_{4}(0,1)} \leq C\left\|\partial_{y} w_{n}(y, t)\right\|_{H^{1}(0,1)}^{1 / 2}\left\|\partial_{y} w_{n}(y, t)\right\|_{L_{2}(0,1)}^{1 / 2}, \forall \partial_{y} w_{n}(y, t) \in H^{1}(0,1),
$$


from (4.8), we obtain

$$
\begin{gathered}
\alpha_{2}\left|\left(w_{n}(y, t) \partial_{y} w_{n}(y, t), \partial_{y}^{2} w_{n}(y, t)\right)\right| \leq \\
\leq C\left\|w_{n}(y, t)\right\|_{L_{4}(0,1)}\left\|\partial_{y} w_{n}(y, t)\right\|_{H^{1}(0,1)}^{3 / 2}\left\|\partial_{y} w_{n}(y, t)\right\|_{L_{2}(0,1)}^{1 / 2} \leq \\
\leq \frac{\beta_{1}}{8}\left\|\partial_{y}^{2} w_{n}(y, t)\right\|_{L_{2}(0,1)}^{2}+\left[\frac{\beta_{1}}{8}+C_{2}\left\|w_{n}(y, t)\right\|_{L_{4}(0,1)}^{4}\right]\left\|\partial_{y} w_{n}(y, t)\right\|_{L_{2}(0,1)}^{2} .
\end{gathered}
$$

Here, we have used Young's inequality (4.5), where

$$
A=\left\|\partial_{y} w_{n}(y, t)\right\|_{H^{1}(0,1)}^{3 / 2}, \quad B=C\left\|w_{n}(y, t)\right\|_{L_{4}(0,1)}\left\|\partial_{y} w_{n}(y, t)\right\|_{L_{2}(0,1)}^{1 / 2}, \quad a=\frac{\beta_{1}}{6}, \quad p=\frac{4}{3}, \quad q=4 .
$$

Further, for the last two summands from (4.4) we will have:

$$
\begin{aligned}
\gamma_{1}\left|\left(\partial_{y} w_{n}(y, t), \partial_{y}^{2} w_{n}(y, t)\right)\right| & \leq \frac{\beta_{1}}{8}\left\|\partial_{y}^{2} w_{n}(y, t)\right\|_{L_{2}(0,1)}^{2}+C_{3}\left\|\partial_{y} w_{n}(y, t)\right\|_{L_{2}(0,1)}^{2} \\
\left|\left(g(y, t), \partial_{y}^{2} w_{n}(y, t)\right)\right| & \leq \frac{\beta_{1}}{4}\left\|\partial_{y}^{2} w_{n}(y, t)\right\|_{L_{2}(0,1)}^{2}+C_{4}\|g(y, t)\|_{L_{2}(0,1)}^{2} .
\end{aligned}
$$

Taking into account inequalities (4.6)-(4.11), integrating (4.4) from 0 to $t$, we get

$$
\begin{gathered}
\left\|\partial_{y} w_{n}(y, t)\right\|_{L_{2}(0,1)}^{2}+\beta_{1} \int_{0}^{t}\left\|\partial_{y}^{2} w_{n}(y, \tau)\right\|_{L_{2}(0,1)}^{2} d \tau \leq A_{4}\|g(y, t)\|_{L_{2}(Q)}^{2}+ \\
+\int_{0}^{t} A_{5}(\tau)\left\|\partial_{y} w_{n}(y, \tau)\right\|_{L_{2}(0,1)}^{2} d \tau+2 C_{0} \int_{0}^{t}\left|w_{n}(0, t)\right|^{3} d t+2 C_{1} \int_{0}^{t}\left|w_{n}(1, t)\right|^{3} d t+2\left(D_{0}+D_{1}\right),
\end{gathered}
$$

where

$$
A_{4}=2 C_{4}, \quad A_{5}(t)=\frac{1}{2}+\frac{\beta_{1}}{4}+2 C_{2}\left\|w_{n}(y, t)\right\|_{L_{4}(0,1)}^{4}+2 C_{3} .
$$

Let us estimate the last two integral summands from (4.12). By (4.6)-(4.7), we have

$$
\begin{aligned}
& 2 C_{0} \int_{0}^{t}\left|w_{n}(0, t)\right|^{3} d t \leq \frac{C_{0}}{4} \int_{0}^{t}\left\|\partial_{y} w_{n}(y, t)\right\|_{L_{2}(0,1)}^{2} d t+2 D_{0} T, \\
& 2 C_{1} \int_{0}^{t}\left|w_{n}(0, t)\right|^{3} d t \leq \frac{C_{1}}{4} \int_{0}^{t}\left\|\partial_{y} w_{n}(y, t)\right\|_{L_{2}(0,1)}^{2} d t+2 D_{1} T,
\end{aligned}
$$

Thus, (4.12) takes the form:

$$
\begin{aligned}
& \left\|\partial_{y} w_{n}(y, t)\right\|_{L_{2}(0,1)}^{2}+\beta_{1} \int_{0}^{t}\left\|\partial_{y}^{2} w_{n}(y, \tau)\right\|_{L_{2}(0,1)}^{2} d \tau \leq A_{4}\|g(y, t)\|_{L_{2}(Q)}^{2}+ \\
& +\int_{0}^{t}\left[A_{5}(\tau)+\frac{C_{0}+C_{1}}{4}\right]\left\|\partial_{y} w_{n}(y, \tau)\right\|_{L_{2}(0,1)}^{2} d \tau+2\left(D_{0}+D_{1}\right)(1+T),
\end{aligned}
$$

From inequality (4.13) in the same way as in the proof of Lemma 4.1, we obtain the desired estimate (4.3). Lemma 4.2 is completely proved.

Lemma 4.3. For positive a constant $K_{3}$ independent of $n$, at all $t \in(0, T]$ the following inequality holds:

$$
\left\|\partial_{t} w_{n}(y, t)\right\|_{L_{2}\left(Q_{y t}\right)}^{2} \leq K_{3}
$$


Proof. Let us write down equation (1.1) for the approximate solution $w_{n}(y, t)$ :

$$
\partial_{t} w_{n}+\alpha(t) w_{n} \partial_{y} w_{n}-\beta(t) \partial_{y}^{2} w_{n}+\gamma(y, t) \partial_{y} w_{n}=g .
$$

From equation (4.15), we obtain

$$
\left\|\partial_{t} w_{n}\right\|_{L_{2}\left(Q_{y t}\right)} \leq \alpha_{2}\left\|w_{n} \partial_{y} w_{n}\right\|_{L_{2}\left(Q_{y t}\right)}+\beta_{2}\left\|\partial_{y}^{2} w_{n}\right\|_{L_{2}\left(Q_{y t}\right)}+\gamma_{1}\left\|\partial_{y} w_{n}\right\|_{L_{2}\left(Q_{y t}\right)}+\|g\|_{L_{2}\left(Q_{y t}\right)} .
$$

According to embedding $H^{1}(0,1) \hookrightarrow L_{\infty}(0,1)$ inequality $\left\|w_{n}\right\|_{L_{\infty}(0,1)} \leq B\left\|w_{n}\right\|_{H^{1}(0,1)}$ holds. Hence, considering Lemmas 4.1 and 4.2 , we obtain

$$
\begin{gathered}
\left\|w_{n} \partial_{y} w_{n}\right\|_{L_{2}\left(Q_{y t}\right)}^{2} \leq \int_{0}^{T}\left\|w_{n}(y, t)\right\|_{L_{\infty}(0,1)}^{2}\left\|\partial_{y} w_{n}(y, t)\right\|_{L_{2}(0,1)}^{2} d t \leq \\
\leq B\left\|\partial_{y} w_{n}(y, t)\right\|_{L_{\infty}\left(0, T ; L_{2}(0,1)\right)}^{2} \int_{0}^{T}\left\|w_{n}(y, t)\right\|_{H^{1}(0,1)}^{2} d t \leq B K_{2} K_{1}(1+T),
\end{gathered}
$$

where $B$ is a constant of embedding $H^{1}(0,1) \hookrightarrow L_{\infty}(0,1), K_{1}$ and $K_{2}$ are constants from Lemmas 4.1 and 4.2 , respectively.

Estimate (4.14) follows from (4.16), (4.17) and from the statement of Lemmas 4.1 and 4.2, Lemma 4.3 is completely proved.

\section{Unique solvability of initial boundary value problem (1.1)-(1.3)}

Lemmas 4.1-4.3 show that the sequence of Galerkin approximations $\left\{w_{n}(y, t), n=1,2,3, \ldots\right\}$ is bounded in space $L_{\infty}\left(0, T ; H^{1}(0,1)\right) \cap L_{2}\left(0, T ; H^{2}(0,1)\right)$, and the sequence $\left\{\partial_{t} w_{n}(y, t), n=1,2,3, \ldots\right\}$ is bounded in $L_{2}\left(0, T ; L_{2}(0,1)\right)$.

Thus, we can extract a weakly convergent subsequence (we preserve the notation of the index $n$ for the subsequence):

$$
\begin{gathered}
w_{n}(y, t) \rightarrow w(y, t) \text { weakly in } L_{2}\left(0, T ; H^{2}(0,1)\right) \cap H^{1}\left(0, T ; L_{2}(0,1)\right), \\
w_{n}(y, t) \rightarrow w(y, t) \text { strongly in } L_{2}\left(0, T ; L_{2}(0,1)\right) \text { and almost everywhere in } Q_{y t},
\end{gathered}
$$

Lemma 5.1. Let conditions (1.4)-(1.5) be satisfied and $g \in L_{2}\left(Q_{y t}\right)$. Then initial boundary value problem (1.1)-(1.3) has a weak solution in space $H^{2,1}\left(Q_{y t}\right)$.

Proof. Let $\varphi(t) \in \mathcal{D}((0, T))$, i.e. from the class of infinitely differentiable finite functions. We introduce the notation $v_{j}(y, t)=\varphi(t) Y_{j}(y)$, where $Y_{j}(y) \in H^{1}(0,1)$. Now, by multiplying integral identity (3.4) by the function $\varphi(t) \in \mathcal{D}((0, T))$ and integrating the result obtained with respect to $t$ from 0 to $T$, we obtain

$$
\begin{aligned}
\int_{0}^{T} \int_{0}^{1}\left[\partial_{t} w_{n}+\alpha(t) w_{n} \partial_{y} w_{n}-\beta(t) \partial_{y}^{2} w_{n}+\gamma(y, t) \partial_{y} w_{n}\right] v_{j} d y d t+ \\
+\int_{0}^{T}\left[\beta(t) \partial_{y} w_{n}(1, t)-\frac{\alpha(t)}{3} w_{n}^{2}(1, t)\right] v_{j}(1, t) d t+ \\
+\int_{0}^{T}\left[-\beta(t) \partial_{y} w_{n}(0, t)+\frac{\alpha(t)}{3} w_{n}^{2}(0, t)\right] v_{j}(0, t) d t= \\
=\int_{0}^{T} \int_{0}^{1} g v_{j} d y d t, \quad \forall \varphi(t) \in \mathcal{D}((0, T)), \forall j=1, \ldots, n .
\end{aligned}
$$


Since $\mathcal{D}\left((0, T) ; H^{1}(0,1)\right)$ is dense in $L_{2}\left(0, T ; H^{1}(0,1)\right)$, then integral identity $(5.3)$ can be rewritten as

$$
\begin{aligned}
\int_{0}^{T} \int_{0}^{1}\left[\partial_{t} w_{n}+\alpha(t) w_{n} \partial_{y} w_{n}-\beta(t) \partial_{y}^{2} w_{n}+\gamma(y, t) \partial_{y} w_{n}\right] v d y d t+ \\
+\int_{0}^{T}\left[\beta(t) \partial_{y} w_{n}(1, t)-\frac{\alpha(t)}{3} w_{n}^{2}(1, t)\right] v(1, t) d t+ \\
+\int_{0}^{T}\left[-\beta(t) \partial_{y} w_{n}(0, t)+\frac{\alpha(t)}{3} w_{n}^{2}(0, t)\right] v(0, t) d t= \\
=\int_{0}^{T} \int_{0}^{1} g v d y d t, \forall v(y, t) \in L_{2}\left(0, T ; H^{1}(0,1)\right) .
\end{aligned}
$$

In integral identity (5.4), we pass to the limit as $n \rightarrow \infty$. In the expressions corresponding to the linear summands of equation (1.1) and boundary conditions (1.2), passing to the limit is carried out according to relation (5.1). As for the nonlinear summands, here, we have the following:

$$
\begin{gathered}
\int_{0}^{T} \int_{0}^{1} \alpha(t) w_{n}(y, t) \partial_{y} w_{n}(y, t) v(y, t) d y d t=\int_{0}^{T} \alpha(t) \int_{0}^{1}\left[w_{n}(y, t)-w(y, t)\right] \partial_{y} w_{n}(y, t) v(y, t) d y d t+ \\
\quad+\int_{0}^{T} \alpha(t) \int_{0}^{1} w(y, t) \partial_{y} w_{n}(y, t) v(y, t) d y d t \rightarrow \int_{0}^{T} \alpha(t) \int_{0}^{1} w(y, t) \partial_{y} w(y, t) v(y, t) d y d t
\end{gathered}
$$

since according to (5.2) and (5.1) the following limit relation holds

$$
\int_{0}^{T} \alpha(t) \int_{0}^{1}\left[w_{n}(y, t)-w(y, t)\right] \partial_{y} w_{n}(y, t) v(y, t) d y d t \rightarrow 0 .
$$

Further, according to (5.4) and (5.2) similarly to the previous one, we will have

$$
\begin{gathered}
\int_{0}^{T} w_{n}(1, t) w_{n}(1, t) v(1, t) d t=\int_{0}^{T}\left[w_{n}(1, t)-w(1, t)\right] w_{n}(1, t) v(1, t) d t+ \\
+\int_{0}^{T} w(1, t) w_{n}(1, t) v(1, t) d t \rightarrow \int_{0}^{T} w^{2}(1, t) v(1, t) d t, \\
\int_{0}^{T} w_{n}(0, t) w_{n}(0, t) v(0, t) d t=\int_{0}^{T}\left[w_{n}(0, t)-w(0, t)\right] w_{n}(0, t) v(0, t) d t+ \\
+\int_{0}^{T} w(0, t) w_{n}(0, t) v(0, t) d t \rightarrow \int_{0}^{T} w^{2}(0, t) v(0, t) d t .
\end{gathered}
$$

So, by passing to the limit at $n \rightarrow \infty$ in integral identity (5.4), taking into account limit relations (5.5)-(5.7), as well as in initial condition (3.3), we get

$$
\int_{0}^{T} \int_{0}^{1}\left[\partial_{t} w+\alpha(t) w \partial_{y} w-\beta(t) \partial_{y}^{2} w+\gamma(y, t) \partial_{y} w\right] v d y d t+
$$




$$
\begin{gathered}
+\int_{0}^{T}\left[\beta(t) \partial_{y} w(1, t)-\frac{\alpha(t)}{3} w^{2}(1, t)\right] v(1, t) d t+ \\
+\int_{0}^{T}\left[-\beta(t) \partial_{y} w(0, t)+\frac{\alpha(t)}{3} w^{2}(0, t)\right] v(0, t) d t= \\
=\int_{0}^{T} \int_{0}^{1} g v d y d t, \forall v(y, t) \in L_{2}\left(0, T ; H^{1}(0,1)\right) . \\
\int_{0}^{1} w(y, 0) \psi(y) d y=0, \forall \psi \in L_{2}(0,1) .
\end{gathered}
$$

Note that integral identity (5.8) is also valid for any test function $v(y, t) \in L_{2}\left(0, T ; H_{0}^{1}(0,1)\right) \subset L_{2}\left(0, T ; H^{1}(0,1)\right)$, i.e. we have

$$
\int_{0}^{T} \int_{0}^{1}\left[\partial_{t} w+\alpha(t) w \partial_{y} w-\beta(t) \partial_{y}^{2} w+\gamma(y, t) \partial_{y} w-g\right] v d y d t=0, \quad \forall v(y, t) \in L_{2}\left(0, T ; H_{0}^{1}(0,1)\right) .
$$

Further, returning to (5.8) and taking into account that traces $v(1, t)$ and $v(0, t)$ from $L_{2}(0, T)$ of test function $v \in L_{2}\left(0, T ; H^{1}(0,1)\right)$ are independent of each other and are arbitrary, in this case the following identities

$$
\begin{aligned}
& \int_{0}^{T}\left[\beta(t) \partial_{y} w(1, t)-\frac{\alpha(t)}{3} w^{2}(1, t)\right] \psi_{1}(t) d t=0, \quad \forall \psi_{1}(t) \in L_{2}(0, T), \\
& \int_{0}^{T}\left[-\beta(t) \partial_{y} w(0, t)+\frac{\alpha(t)}{3} w^{2}(0, t)\right] \psi_{0}(t) d t=0, \quad \forall \psi_{0}(t) \in L_{2}(0, T),
\end{aligned}
$$

follow from (5.8), that is, the integrands in square brackets from (5.10)-(5-12) define zero functionals over spaces $L_{2}\left(0, T ; H_{0}^{1}(0,1)\right)$ and $L_{2}(0, T)$, and belong to spaces $0 \in L_{2}\left(0, T ; H^{-1}(0,1)\right) \subset \mathcal{D}^{\prime}\left(Q_{y t}\right)$ and $0 \in L_{2}(0, T) \subset$ $\mathcal{D}^{\prime}((0, T))$. Thus, from (5.10)-(5.12) we obtain that the weak limit function $w(y, t)$ satisfies equation (1.1) and boundary conditions (1.2), and from (5.9), it follows that it satisfies initial condition (1.3). This completes the proof of Lemma 5.1.

Lemma 5.2. Under the conditions of Lemma 5.1 the solution $w \in H^{2,1}\left(Q_{y t}\right)$ of initial boundary value problem (1.1)-(1.3) is unique.

Proof. Let initial boundary value problem (1.1)-(1.3) have two different solutions $w^{(1)}(y, t)$ and $w^{(2)}(y, t)$. Then their difference $w(y, t)=w^{(1)}(y, t)-w^{(2)}(y, t)$ will satisfy the following homogeneous problem:

$$
\begin{gathered}
\partial_{t} w+\alpha(t) w \partial_{y} w^{(1)}+\alpha(t) w^{(2)} \partial_{y} w-\beta(t) \partial_{y}^{2} w=0, \\
{\left.\left[\frac{\alpha(t)}{3} w\left(w^{(1)}+w^{(2)}\right)-\beta(t) \partial_{y} w\right]\right|_{y=0}=0} \\
{\left.\left[\frac{\alpha(t)}{3} w\left(w^{(1)}+w^{(2)}\right)-\beta(t) \partial_{y} w\right]\right|_{y=1}=0 .}
\end{gathered}
$$

According to Lemmas 4.1 and 4.2 we have

$$
w^{(i)}(y, t) \in L_{\infty}\left(0, T ; H^{1}(0,1)\right) \cap L_{2}\left(0, T ; H^{2}(0,1)\right), i=1,2 .
$$

Multiplying equation (5.13) by function $w(y, t)$ scalarly in $L_{2}(0,1)$ and taking into account (5.14)-(5.16), we obtain

$$
\frac{1}{2} \frac{d}{d t}\|w(y, t)\|_{L_{2}(0,1)}^{2}+\beta_{1}\left\|\partial_{y} w(y, t)\right\|_{L_{2}(0,1)}^{2} \leq \frac{\alpha(t)}{3}|w(1, t)|^{2}\left[w^{(1)}(1, t)+w^{(2)}(1, t)\right]+
$$




$$
+\frac{\alpha(t)}{3}|w(0, t)|^{2}\left[w^{(1)}(0, t)+w^{(2)}(0, t)\right]-\alpha(t) \int_{0}^{1}\left[w \partial_{y} w^{(1)}+w^{(2)} \partial_{y} w\right] d y .
$$

Now, we estimate the right-hand side of (5.17). According to (5.16) and by Lemma 4.1, we have:

$$
\begin{gathered}
\frac{\alpha(t)}{3}\left[w^{(1)}(1, t)+w^{(2)}(1, t)\right]|w(1, t)|^{2} \leq \\
\leq \frac{\alpha_{2}}{3}\left[\left\|w^{(1)}(1, t)\right\|_{L_{\infty}(0, T)}+\left\|w^{(2)}(1, t)\right\|_{L_{\infty}(0, T)}\right]|w(1, t)|^{2} \leq C_{1}|w(1, t)|^{2}, \\
\frac{\alpha(t)}{3}\left[w^{(1)}(0, t)+w^{(2)}(0, t)\right]|w(0, t)|^{2} \leq \\
\leq \frac{\alpha_{2}}{3}\left[\left\|w^{(1)}(0, t)\right\|_{L_{\infty}(0, T)}+\left\|w^{(2)}(0, t)\right\|_{L_{\infty}(0, T)}\right]|w(0, t)|^{2} \leq C_{2}|w(0, t)|^{2},
\end{gathered}
$$

Further, we have

$$
\begin{gathered}
\alpha(t) \int_{0}^{1}\left[w^{2} \partial_{y} w^{(1)}+w^{(2)} w \partial_{y} w\right] d y=\alpha(t)\left[|w(1, t)|^{2} w^{(1)}(1, t)-|w(0, t)|^{2} w^{(1)}(0, t)\right]+ \\
+\alpha(t) \int_{0}^{1}\left[-2 w^{(1)} w \partial_{y} w+w^{(2)} w \partial_{y} w\right] d y \leq C_{3}|w(1, t)|^{2}+C_{4}|w(0, t)|^{2}+ \\
+\frac{\alpha_{2}^{2}}{\beta_{1}}\left[2\left\|w^{(1)}\right\|_{L_{\infty}\left(Q_{y^{t}}\right)}+\left\|w^{(2)}\right\|_{L_{\infty}\left(Q_{y t}\right)}\right]^{2}\|w\|_{L_{2}(0,1)}^{2}+\frac{\beta_{1}}{4}\left\|\partial_{y} w\right\|_{L_{2}(0,1)}^{2} \leq \\
\leq C_{3}|w(1, t)|^{2}+C_{4}|w(0, t)|^{2}+C_{5}\|w(y, t)\|_{L_{2}(0,1)}^{2}+\frac{\beta_{1}}{4}\left\|\partial_{y} w\right\|_{L_{2}(0,1)}^{2} .
\end{gathered}
$$

We need the following estimates

$$
\begin{gathered}
\left(C_{1}+C_{3}\right)|w(1, t)|^{2} \leq\left(C_{1}+C_{3}\right)\|w(y, t)\|_{L_{\infty}(0,1)}^{2} \leq \\
\leq\left(C_{1}+C_{3}\right) B\|w(y, t)\|_{H^{1}(0,1)}\|w(y, t)\|_{L_{2}(0,1)} \leq \\
\leq \frac{\beta_{1}}{8}\left\|\partial_{y} w(y, t)\right\|_{L_{2}(0,1)}^{2}+\left[\frac{\beta_{1}}{8}+\frac{2\left(C_{1}+C_{3}\right)^{2} B^{2}}{\beta_{1}}\right]\|w(y, t)\|_{L_{2}(0,1)}^{2}, \\
\left(C_{2}+C_{4}\right)|w(0, t)|^{2} \leq\left(C_{2}+C_{4}\right)\|w(y, t)\|_{L_{\infty}(0,1)}^{2} \leq \\
\leq\left(C_{2}+C_{4}\right) B\|w(y, t)\|_{H^{1}(0,1)}\|w(y, t)\|_{L_{2}(0,1)} \leq \\
\leq \frac{\beta_{1}}{8}\left\|\partial_{y} w(y, t)\right\|_{L_{2}(0,1)}^{2}+\left[\frac{\beta_{1}}{8}+\frac{2\left(C_{2}+C_{4}\right)^{2} B^{2}}{\beta_{1}}\right]\|w(y, t)\|_{L_{2}(0,1)}^{2},
\end{gathered}
$$

where $B$ is the norm of the embedding operator $H^{1}(0,1) \hookrightarrow L_{\infty}(0,1)$.

Based on relations (5.17)-(5.22) we establish

$$
\begin{gathered}
\frac{d}{d t}\|w(y, t)\|_{L_{2}(0,1)}^{2}+\beta_{1}\left\|\partial_{y} w(y, t)\right\|_{L_{2}(0,1)}^{2} \leq \\
\leq\left[\frac{\beta_{1}}{2}+\frac{4 B^{2}}{\beta_{1}}\left(\left(C_{1}+C_{3}\right)^{2}+\left(C_{2}+C_{4}\right)^{2}\right)+2 C_{5}\right]\|w(y, t)\|_{L_{2}(0,1)}^{2}, \quad \forall t \in(0, T] .
\end{gathered}
$$

Hence, applying Gronwall's inequality, we obtain:

$$
\|w(y, t)\|_{L_{2}(0,1)}^{2} \equiv 0, \forall t \in(0, T] .
$$

This means that $w^{(1)}(y, t) \equiv w^{(2)}(y, t)$ in $L_{2}\left(Q_{y t}\right)$, i.e. the solution to initial boundary value problem (1.1)(1.3) can be only one. Lemma 5.2 is completely proved.

From the statements of Lemmas 5.1 and 5.2 follows the validity of Theorem 1.1.

Thus, we have proved the main result of our work - Theorem 1.1. 


\section{Conclusion}

In this paper, we have established a theorem on the unique solvability in Sobolev classes of a Neumann-type boundary value problem for the Burgers equation in a rectangular domain. The established results can be useful in the problems of modeling (a) nonlinear thermal fields in high voltage contact devices, (b) nonlinear processes of diffusion and propagation of foreign inclusions in the flows of water and atmospheric areas, etc.

\section{Acknowledgments}

This research is funded by the Science Committee of the Ministry of Education and Science of the Republic of Kazakhstan (Grant No. AP08855372, 2020-2022).

\section{References}

1 Benia Y. Existence of solutions to Burgers equations in domains that can be transformed into rectangles / Y. Benia, B.K. Sadallah // Electronic Journal of Differential Equations. - 2016. - 157. - P. 1-13.

2 Benia Y. Existence of solutions to Burgers equations in a non-parabolic domain / Y. Benia, B.K. Sadallah // Electronic Journal of Differential Equations. - 2018. - 20. - P. 1-13.

3 Jenaliyev M.T. On Solonnikov-Fasano Problem for the Burgers Equation / M.T. Jenaliyev, M.I. Ramazanov, A.A. Assetov // Bulletin of the Karaganda University. Mathematics Series. - 2020. - 2(98). P. 69-81.

4 Jenaliyev M.T. On solvability of one nonlinear boundary value problem of heat conductivity in degenerating domains / M.T. Jenaliyev, K.B. Imanberdiyev, A.S. Kassymbekova, M.G. Yergaliyev // Kazakh Mathematical Journal. - 2020. - 20(1). - P. 67-83.

5 Burgers J.M. The nonlinear diffusion equation. Asymptotic solutions and statistical problems / J.M. Burgers // Advances in Difference Equations. - Boston, USA: D. Reidel Publishing Company, DordrechtHolland, 1974. - $174 \mathrm{p}$.

6 Вишик М.И. Математические задачи статистической гидромеханики / М.И. Вишик, А.В. Фурсиков. - М.: Наука, 1980. - 440 c.

7 Farina A. Non-isothermal injection moulding with resin cure and perform deformability / A. Farina, L. Preziosi // Composites/A. - 2000. - 31. - P. 1355-1372.

8 Nouri Z. N wave and periodic wave solutions for Burgers equations / Z. Nouri, S. Bendaas, H.E. Kadem // International Journal of Analysis and Applications. - 2020. - 18. - №2. - P. 304-318.

9 Yang X.-J. A new fractal nonlinear Burgers' equation arising in the acoustic signals propagation / X.-J. Yang, J.A. Tenreiro Machado // Math. Meth. Appl. Sci. - 2019. - 42. - №18. - P. 7539-7544.

10 Selmi R. Well-posedness to 3D Burgers' equation in critical Gevrey Sobolev spaces / R. Selmi, A. Chaabani // Archiv der Mathematik. - 2019. - 112. - №6. - P. 661-672.

11 Molinet L. On well-posedness for some dispersive perturbations of Burgers' equation / L. Molinet, D. Pilod, S. Vento // Annales De L Institute Henri Poincare - Analyse Non Lineaire. - 2018. - 35. - №7. - P. 1719-1756.

12 Rottmann-Matthes J. Freezing similarity solutions in the multidimensional Burgers equation / J. Rottmann-Matthes // Nonlinearity. - 2017. - 30. - №12. - P. 4558-4586.

13 Zhu N. On the Boussinesq-Burgers equations driven by dynamic boundary conditions / N. Zhu, Zh. Liu, K. Zhao // Journal of Differential Equations. - 2018. — 264. — №3. - P. 2287-2309.

14 Ким Е.И. Математические модели тепловых процессов в электрических контактах / Е.И. Ким, В.Т. Омельченко, С.Н. Харин. - Алма-Ата: Наука, 1977. - 236 с.

15 Митропольский Ю.А. Задачи со свободными границами для нелинейного эволюционного уравнения в проблемах металлургии, медицины, экологии / Ю.А. Митропольский, А.А. Березовский, Т.А. Плотницкий // Укр. мат. журн. - 1992. - 44. - № 1. - С. 67-75.

16 Веригин Н.Н. Об одном классе гидромеханических задач для областей с подвижными границами / Н.Н. Веригин // Динамика жидкости со свободными границами. СО АН СССР. - 1980. - 46. C. $23-32$. 
17 Карташов Э.М. Проблема теплового удара в области с движущейся границей на основе новых интегральных соотношений / Э.М. Карташов // Изв. РАН. - Энергетика. - 1997. - 4. - С. $122-137$.

18 Riesz F. Lecons d'Analyse Fonctionelle / F. Riesz, Bela Sz.-Nagy // Akademiai Kiado, Budapest. - 1972.

19 Adams R.A. Sobolev spaces (2-nd Edition) / R.A. Adams, J.J.F. Fournier. - Elsevier, Amsterdam. 2003. - 140. - P. 320 .

\author{
М.Т. Жиенәлиев ${ }^{1}$, М.Ғ. Ерғалиев ${ }^{1}$, Ә.Ә. Әсетов ${ }^{2}$, А.Қ. Қалибекова ${ }^{1}$ \\ ${ }^{1}$ Математика және математикалық, модельдеу институты, Алматы, Қазақстан; \\ ${ }^{2}$ Академик Е.А. Бөкетов атындавы Қараванды университеті, Қараванды, Қазақстан
}

\title{
Тікбұрышты облыстағы Бюргерс теңдеуі үшін бір бастапқы шекаралық есеп туралы
}

\begin{abstract}
Тікбұрышты облыстағы Бюргерс теңдеуінің кейбір бастапқы шекаралық есептері қарастырылған, бір мағынада оны моделді ретінде қабылдауға болады. Шындығында, мұндай мәселе көбінесе қозғалатын шекаралары бар облыстардағы Бюргерс теңдеулерін зерттеу кезінде туындайды. Айтылғанды растау үшін, мыналарға жүгінуге болады: [1] және [2] жұмыстарға. Функционалдық талдау әдістерін, ФаэдоГалеркин және априорлық бағалау әдістерін қолдана отырып, Соболев кеңістігінде және тікбұрышты облыста Нейманн типіндегі сызықтық емес шекаралық шарттармен берілген Бюргерс теңдеуі үшін бастапқы шекаралық есептің дұрыс қойылғандығы көрсетілген.
\end{abstract}

Kiлm сөздер: Бюргерс теңдеуі, шекаралық есеп, Соболевтік кеңістік, тікбұрышты облыс, Галеркин әдісі, априорлық бағалаулар.

\author{
М.Т. Дженалиев ${ }^{1}$, М.Г. Ергалиев ${ }^{1}$, А.А. Асетов ${ }^{2}$, А.К. Калибекова ${ }^{1}$ \\ ${ }^{1}$ Институт математики и математического моделирования, Алматы, Казахстан; \\ ${ }^{2}$ Карагандинский университет имени академика Е.А. Букетова, Караганда, Казахстан
}

\section{Об одной начальной граничной задаче для уравнения Бюргерса в прямоугольной области}

В статье рассмотрена некоторая начально-граничная задача для уравнения Бюргерса в прямоугольной области, которую в известном смысле можно принять за модельную. Дело в том, что такая проблема часто возникает при изучении уравнения Бюргерса в областях с движущимися границами. В подтверждение сказанного можно сослаться на работы [1 и 2]. С помощью методов функционального анализа, априорных оценок и Фаэдо-Галеркина в пространствах Соболева и в прямоугольной области авторами показа на корректность начально-граничной задачи для уравнения Бюргерса с нелинейными граничными условиями типа Неймана.

Ключевые слова: уравнение Бюргерса, граничная задача, пространство Соболева, прямоугольная область, метод Галеркина, априорные оценки.

\section{References}

1 Benia, Y., \& Sadallah, B.-K. (2016). Existence of solutions to Burgers equations in domains that can be transformed into rectangles. Electronic Journal of Differential Equations, 157, 1-13.

2 Benia, Y., \& Sadallah, B.-K.(2018). Existence of solutions to Burgers equations in a non-parabolic domain. Electronic Journal of Differential Equations, 20, 1-13. 
3 Jenaliyev, M.T., Ramazanov, M.I., \& Assetov, A.A. (2020). On Solonnikov-Fasano Problem for the Burgers Equation. Bulletin of the Karaganda University, Mathematics Series, 2(98), 69-81.

4 Jenaliyev, M.T., Imanberdiyev, K.B., Kassymbekova, A.S., \& Yergaliyev, M.G. (2020). On solvability of one nonlinear boundary value problem of heat conductivity in degenerating domains. Kazakh Mathematical Journal, 20(1), 67-83.

5 Burgers, J.M. (1974). The nonlinear diffusion equation. Asymptotic solutions and statistical problems. Advances in Difference Equations. Boston, USA: D. Reidel Publishing Company, Dordrecht-Holland, 174.

6 Vishik, M.I., \& Fursikov, A.V. (1980). Matematicheskie zadachi statisticheskoi gidrodinamiki [Mathematical problems of statistical hydromechanics]. Moscow: Nauka [in Russian].

7 Farina, A., \& Preziosi, L. (2000). Non-isothermal injection moulding with resin cure and perform deformability. Composites/A, 31, 1355-1372.

8 Nouri, Z., Bendaas, S., \& Kadem, H.E. (2020). N wave and periodic wave solutions for Burgers equations. International Journal of Analysis and Applications, 18(2), 304-318.

9 Yang, X.-J., \& Tenreiro Machado, J.A. (2019). PA new fractal nonlinear Burgers' equation arising in the acoustic signals propagation. Math. Meth. Appl. Sci., 42, 18, 7539-7544.

10 Selmi, R., \& Chaabani, A. (2019). Well-posedness to 3D Burgers' equation in critical Gevrey Sobolev spaces. Archiv der Mathematik, 112(6), 661-672.

11 Molinet, L., Pilod, D., \& Vento, S. (2018). On well-posedness for some dispersive perturbations of Burgers equation. Annales De L Institute Henri Poincare - Analyse Non Lineaire, 35(7), 1719-1756.

12 Rottmann-Matthes, J. (2017). Freezing similarity solutions in the multidimensional Burgers equation. Nonlinearity, 30, 12, 4558-4586.

13 Zhu, N., Liu, Zh., \& Zhao, K. (2018). On the Boussinesq-Burgers equations driven by dynamic boundary conditions. Journal of Differential Equations, 264(3), 2287-2309.

14 Kim, E.I., Omelchenko, V.T., \& Kharin, S.N. (1977). Matematicheskie modeli teplovykh protsessov v elektricheskikh kontaktakh [Mathematical models of thermal processes in electrical contacts] Alma-Ata: Nauka [in Russian].

15 Mitropolsky, Yu.A., Berezovsky, A.A., \& Plotnitskiy, T.A. (1992). Zadachi so svobodnymi granitsami dlia nelineinogo evoliutsionnogo uravneniia $\mathrm{v}$ problemakh metallurgii, meditsiny, ekologii [Problems with free boundaries for the nonlinear evolution equation in metallurgy, medicine, and ecology]. Ukrainskii mathematicheskii zhurnal, 44(1), 67-75 [in Russian].

16 Verigin, N.N. (1980). Ob odnom klasse gidromekhanicheskikh zadach dlia oblastei s podvizhnymi granitsami [On one class of hydromechanical problems for domains with moving boundaries]. Fluid dynamics with free boundaries, 46, 23-32 [in Russian].

17 Kartashov, E.M. (1997). Problema teplovogo udara v oblasti s dvizhushcheisia granitsei na osnove novykh integralnykh sootnoshenii [The problem of heat stroke in a domain with a moving boundary based on new integral relations]. Izvestie RAN. Energetics, 4, 122-137 [in Russian].

18 Riesz, F., \& Sz.-Nagy Bela (1972). Lecons d'Analyse Fonctionelle. Akademiai Kiado, Budapest, 448.

19 Adams, R.A., \& Fournier, J.J.F. (2003). Sobolev spaces. Elsevier, Amsterdam, 320. 